\title{
Insights into Binding Affinity of Flavonoid Compounds from Thai Herbs against 2009 H1N1 Hemagglutinin
}

\author{
Wansiri Innok ${ }^{1}$, Thanyada Rungrotmongkol ${ }^{2,3}$ and Panita Kongsune, ${ }^{1, *}$ \\ ${ }^{1}$ Department of Chemistry, Faculty of Science, Thaksin University, Phattalung 93210, Thailand \\ ${ }^{2}$ Structural and Computational Biology Research Unit, Department of Biochemistry, Faculty of Science, \\ Chulalongkorn University, Bangkok 10330, Thailand \\ ${ }^{3}$ Program in Bioinformatics and Computational Biology, Graduate School, Chulalongkorn University, \\ Bangkok 10330, Thailand
}

('Corresponding author's e-mail: dpanita@tsu.ac.th)

Received: 22 December 2020, Revised: 22 May 2021, Accepted: 22 June 2021

\begin{abstract}
Outbreak of influenza virus is one of serious concerns for public health. Hemagglutinin (HA), a spike-shaped glycoprotein on the viral surface, plays an important role during the early stage of influenza infection. In the present work, a set of flavonoids were screened against 2009 H1N1 HA by computational chemistry techniques. Among 35 flavonoids, the docking results showed that the epicatechin gallate (ECG) and puerarin exhibited a good binding affinity towards 2009 H1N1 HA. These 2 compounds were then studied by all-atom molecular dynamics (MD) simulations. The predicted binding free energy of the H1-puerarin complex $(-25.86 \pm 2.92 \mathrm{kcal} / \mathrm{mol})$ was slightly greater than that of H1-ECG $(-22.81 \pm 2.19 \mathrm{kcal} / \mathrm{mol})$, suggesting that the puerarin and ECG could provide similar binding affinity towards $2009 \mathrm{H} 1 \mathrm{HA}$ target. However, the stronger electrostatic energy contribution of $\sim 10$ $\mathrm{kcal} / \mathrm{mol}$ was found in the puerarin binding to $2009 \mathrm{H} 1 \mathrm{~N} 1 \mathrm{HA}$. This molecular information of ligandprotein interaction could be helpful in further drug design and development for influenza treatment.
\end{abstract}

Keywords: Hemagglutinin, Epicatechin gallate, Puerarin, Docking, MD simulation

\section{Introduction}

The influenza viruses affect the millions of people all over the world. Among the three types A, B and $\mathrm{C}$ of influenza viruses, the type $\mathrm{A}$ is responsible for pandemic outbreaks [1]. Hemagglutinin (HA), a spike-shaped glycoprotein extending from the surface of the virus, plays an essential role during the early stage of influenza infection. HA is a homotrimeric envelope glycoprotein which is proteolytically cleaved into 2 functional subunits HA1 and HA2, linked by a disulfide bond [2,3]. HA1 is responsible for host receptor binding, contains terminal sialic acid, while HA2 is related to membrane fusion [4]. Therefore, the HA protein was considered as an attractive target for drug development. The 2 groups of the HA inhibitors (HAIs) are receptor binding inhibitor (RBI) and fusion inhibitor (FI). Up to date, no drug targeting at HA has been approved by the food and drug administration of USA (FDA) [5]. So, it is challenging to discover the effective HA inhibitors against influenza A virus. Blocking the receptor binding domain (RBD) of HA protein with suitable inhibitors could lead to neutralize the influenza virus, resulted in no further viral attachment with the host receptor.

Two classes of anti-influenza drugs approved by FDA including M2 ion channel inhibitors (amantadine and rimantadine) and NA inhibitors (oseltamivir, zanamivir and peramivir) have several limitations in clinical practice, especially the rapid global spread of drug-resistance [6,7]. With the growing problem of resistance, there is an urgent need to focus on new other targets such as the RBD of HA protein. Many natural products showed inhibitory activity on influenza virus. Flavonoids are widely distributed in various plant species, including fruits and vegetables, with one or more hydroxylated aromatic rings in monomer or polymer form [7]. Catechins, such as epicatechin gallate (ECG), epigallocatechin (EGC) and epigallocatechin gallate (EGCG), are polyphenolic compounds of flavonoid derived from the green tea leaves of an evergreen plant showing inhibitory activity of influenza virus [8]. Andrographolide and its derivatives derived from Andrographis paniculata have been used for the treatment of influenza with fever [9], sore throat, chronic cough and other human diseases [10]. The herbs 
such as gallic acid, curcumin and its derivatives and mushroom [11-14], have attracted increasing attention for influenza agents targeting both HA and NA proteins of influenza viruses. Though several in vitro studies showed that natural compounds have anti-influenza activity [7], their inhibition mechanism of action is not known and even more difficult to analyze at the molecular level using experimental methods. Therefore, comprehensive studies are required to determine their molecular interactions.

Recently, several studies have used the molecular docking method to study the interactions and conformations of ligand against HA target $[5,15,16]$, while molecular dynamics (MD) simulations have been applied to study the structural properties of HA-ligand binding [5,6,17]. Herein, we aimed to explore the lead compound from several natural sources for their medicinal potentials as therapeutic agents against influenza. The 35 flavonoid bioactive molecules were selected for screening against HA by molecular docking owing to there are generally regarded as safe by the FDA, hence there are no dosedependent side effects. Moreover, these molecules are obtained from many kinds of Thai herbs such as green tea, andrographis paniculate, gallic acid, curcumin and pueraria. So, the 35 flavonoid bioactive molecules (Figure 1) were used to explore the lead compound for their medicinal potentials as therapeutic agents against influenza. The dynamics information of the screened compounds was then investigated by MD simulations. A detailed understanding of ligand-protein interaction could be helpful in further drug design and development for influenza treatment.

\section{Materials and methods}

\section{System preparation}

The 3-dimensional (3D) structure of the $2009 \mathrm{H} 1 \mathrm{HA}$ protein was retrieved from the Brookhaven Protein Data Bank (PDB) [18] ID 3UBE in which (Resolution: $2.15 \AA$, R-Value Free: 0.252, R-Value Work: 0.193) [19]. The chain A of 3UBE was selected and then the water molecules and were eliminated. All missing hydrogen atoms were added to this protein. The 35 flavonoid compounds were structurally drawn using Gauss View [20] and then were optimized at the Hartree-Fock level with 6-31G* basis function using the Gaussion 03 program [21]. The optimized structures were converted to .pdb format using GaussView [20] for docking study.

\section{Molecular docking}

The Autodock 4.2 program [22] was used to examine the binding affinity of the 35 flavonoid compounds (Figure 1) from Thai Herbs toward the binding site of 2009 HA protein. Partial atomic charges were assigned using the Gasteiger-Marsili method [23] implemented in AutoDock Tools [24]. For structure preparation of $\mathrm{H} 1$ protein and all focused compounds, the protonation of the ionizable amino acids and ligands was assigned at $\mathrm{pH}$ 7.0. Semiflexible docking protocol was set as follows. The H1 protein molecule was kept rigid throughout docking while the ligand compounds were allowed to flexible inside the binding pocket to attain a degree of freedom torsions bridged by the rotational parameter for sampling different binding poses. The PDB of protein and ligands were converted to .pdbqt after initial addition of hydrogen bonds and charges. The cubical grid box of $60 \times 60 \times 60$ size with $0.375 \AA$ was fixed at the active site of the 2009 H1N1 HA protein [Y98, G134, V135, T137, A138, K146, H184, D190, K222, D225, Q226, E227 and G228] [17,25]. Compound sialic acid (SIA) inserted directly into the HA1 subunit binding pocket; therefore, SIA was used as control and was re-docked into the binding site of the HA1 subunit for assessing binding affinity. The binding energy of H1-SIA system was used for filtering flavonoid compounds from Thai Herbs. Autogrid4 parameter was used to attain a rigid grid box. Further to the autogrid 4 and autodock 4 with Lamarckian genetic algorithms and through a protocol with a number of $100 \mathrm{GA}$ runs was used to gain the docking conformations. The conformations that differed by less than $2.0 \AA$ in positional root-mean-square deviation (rmsd) were clustered together. The percentage of possible conformation or dock score of each ligand was reported as \% DS. Other parameters were set as default. After running autogrid and autodock, the possible poses of compounds in HA were obtained. For each compound, the docked conformation with the lowest binding energy and highest ligand-protein interactions was selected. The compounds were ranked according to their docked energies and the top ranked compounds were then chosen for performing molecular dynamics simulations. 


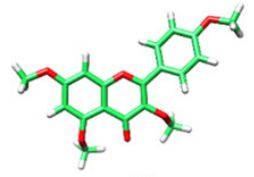

(1)

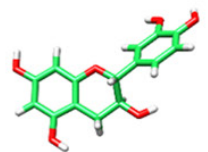

(6)

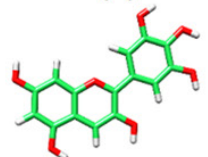

(11)

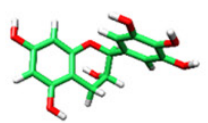

(16)

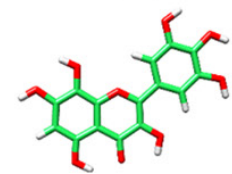

(21)

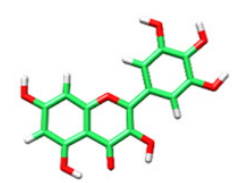

(26)

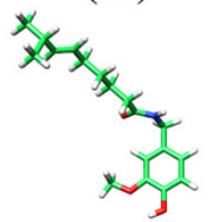

(31)

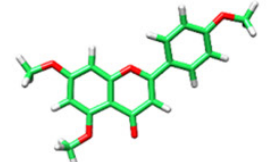

(2)

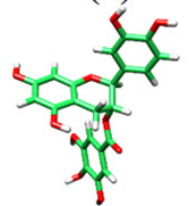

(7)

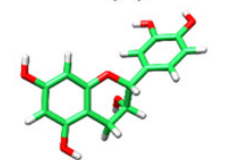

(12)

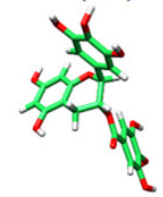

(17)

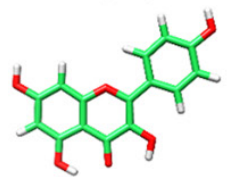

(22)

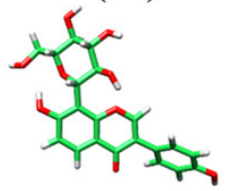

(27)

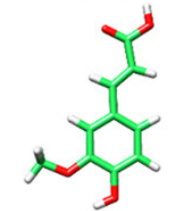

(32)

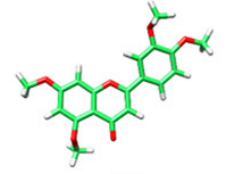

(3)

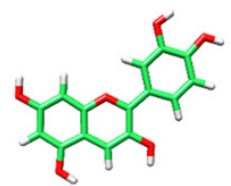

(8)

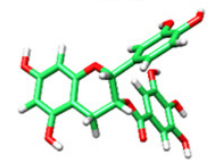

(13)

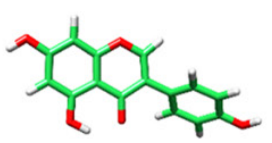

(18)

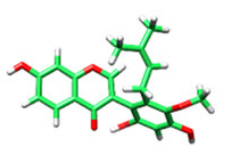

(23)

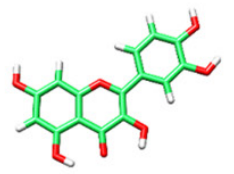

(28)

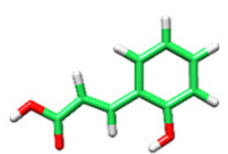

(33)

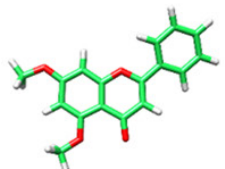

(4)

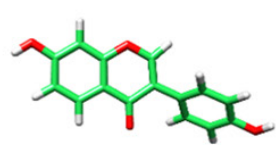

(9)

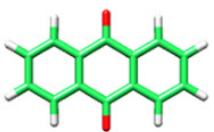

(5)

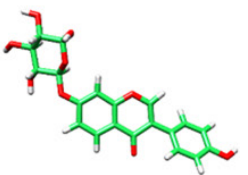

(10)

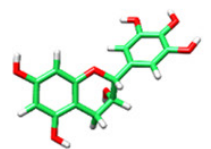

(14)

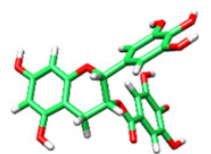

(15)

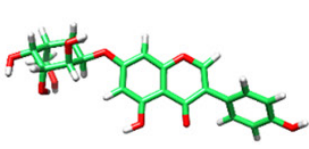

(19)

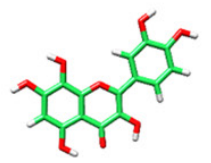

(20)

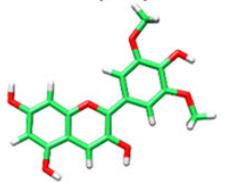

(24)

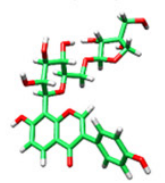

(25)

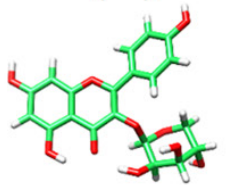

(29)

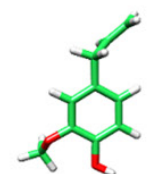

(30)

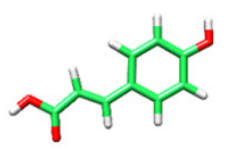

(34)

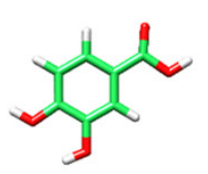

(35)

Figure 1 Structures of optimized geometry of 35 compounds in HF/6-31G* level of theory.

The AutoDock estimates the free energy of binding $(\Delta \mathrm{G})$ based on empirical weighting factors: $\Delta \mathrm{G}=\Delta \mathrm{G}_{\mathrm{vdw}}+\Delta \mathrm{G}_{\text {hbond }}+\Delta \mathrm{G}_{\text {elec }}+\Delta \mathrm{G}_{\mathrm{tor}}+\Delta \mathrm{G}_{\text {sol }}$

where $\Delta \mathrm{G}_{\mathrm{vdw}}, \Delta \mathrm{G}_{\mathrm{hbond}}$ and $\Delta \mathrm{G}_{\text {elec }}$ are the typical molecular mechanics energy terms for van der Waals, hydrogen bonding, and electrostatics interactions, respectively [26]. While $\Delta \mathrm{G}_{\text {tor }}$ characterizes the loss of torsional entropy upon binding and $\Delta \mathrm{G}_{\mathrm{sol}}$ displays the desolvation upon the ligand binding and corresponding hydrophobic effect [27].

\section{Molecular dynamics simulation}

Selected flavonoid candidates in complex with 2009 H1N1 HA were taken for MD simulations to study the ligand-protein dynamics behaviors and interactions, as well as the key binding residues and binding efficiencies. First, this complex was minimized by keeping the ligands and HA fixed. For relaxing the modeled systems, 3 steps of restrained MDs at $298 \mathrm{~K}$ were carried out with the restraining 
factors of $30,20,10$ and $5 \mathrm{~kJ} \mathrm{~mol}^{-1} \AA^{-2}$ for each $500 \mathrm{ps}$. The conformation of the ligand was found to adapt (from the initial model) to fit better with the HA cavity. The last snapshot obtained from the restrained MDs procedure was used as the starting structure of the substrate-enzyme complex for the next MDs with all atoms being allowed to move freely along simulation. The same protocol was employed to build the structural model of each other simulated systems. All calculations were carried out using the AMBER 16 software package [28]. Partial geometric optimization of the hydrogen atoms of ligand was performed at the Hartree-Fock level with 6-31G** basis function using the Gaussion 03 program [21]. Then, single-point calculation was carried out to compute the electrostatic potentials around each compound using the same basis set and level of theory. The electrostatic potential was calculated by RESP [29].

The HA protein were treated by AMBER ff03.r1 force fields [30], while the partial charge generation and assignment of the force field [31] were performed using the Antechamber suite [32]. Protonation of the ionizable amino acids was assigned at $\mathrm{pH} 7.0$ using the PROPKA program [33,34]. Each system was solvated by TIP3P water and the PBC with the NPT ensemble was employed. Energy minimizations and MD simulations were carried out using the SANDER module of AMBER 16 [28]. A Berendsen coupling time of 0.2 ps was used to maintain the temperature and standard pressure of the system [35]. The SHAKE algorithm [36] was applied to constrain all bonds involving hydrogens. The simulation time step of 2 fs was used. All MD simulations were performed with a $10 \AA$ residue-based cutoff for non-bonded interactions and the particle mesh Ewald method was used for an adequate treatment of long-range electrostatic interactions [37]. The MD trajectories were collected every 0.2 ps. Analysis of all MD trajectories, i.e., RMSD, hydrogen bonds etc., were carried out using the ccptraj modules [38] of the AMBER 16. The convergences of energies and global RMSD (root mean square displacement) were used to verify the stability of the systems. Hydrogen bond analysis was performed for identifying the protein-ligand interactions. The MM-PBSA approach was applied to estimate the binding free energy of the systems, using the equilibrium trajectories (100 frames). Water molecules were omitted and replaced by an intrinsic water model, just to focus on the protein-ligand interaction only.

\section{Results and discussion}

\section{Molecular Docking}

The molecular docking was performed using AutoDock program to screen the binding affinity of 35 flavonoid compounds against the $2009 \mathrm{H} 1 \mathrm{HA}$ protein. The 3D structure of these flavonoid compounds from Thai Herbs is shown in Figure 1. The key residues of HA protein can be defined as three important binding regions: 130-loop (residues 133 - 138), 190-helix (residues 190 - 198) and 220-loop (residues 220 - 229) [25,39]. Thus, Y98, G134, V135, T137, A138, K146, H184, D190, K222, D225, Q226, E227 and G228 residues are considered as active residues to bind with flavonoid compounds. The binding energy $(\mathrm{BE}, \mathrm{kcal} / \mathrm{mol})$ and the percentage of possible conformation or dock score (\% DS) of flavonoid compounds from Thai herbs are summarized in Table 1.

The docking program was verified by re-docking of SIA back into HA binding site. The docking model of SIA was well posed in the HA binding site (Figure 2(a)) with the BE and \% DS of -4.68 $\mathrm{kcal} / \mathrm{mol}$ and $99 \%$, suggesting that the setting parameters were suitable for this study. The BE and \% DS of 35 flavonoids compounds were varying from -4.15 to $-7.89 \mathrm{kcal} / \mathrm{mol}$ and 24 to $100 \%$, respectively. On the comparing the binding energy values of all compounds, the top eight flavonoid compounds of the catechin gallate (CG_7), daizin_10, epicatechin gallate (ECG_13), epigallocatechin gallate (EGCG_15), catechin gallate $\left(\mathrm{GC}_{-}^{-} 16\right)$, gallocatechin gallate $\left(\mathrm{GCG}_{-} 17\right)$, mirificin_25 and Puerarin 27 were possess higher binding affinity with the HA1 binding site of $2009 \mathrm{H} 1 \mathrm{HA}$ protein. The BE values of these compounds were $-7.63,-7.42,-7.20,-7.43,-7.25,-7.70,-7.42$ and $-7.89 \mathrm{kcal} / \mathrm{mol}$, respectively when the DS values were $30,94,30,29,61,24,33$ and $46 \%$, respectively. However, the important criteria of strong interaction with key residues in the receptor binding domain (RBD) such as Y98, G134, V135, T137, A138, K146, H183, D190, K222, D225, Q226, E227 and G228 was also considered for finding potential inhibitor. The hydrogen bond interaction and bond distances of 8 flavonoid compounds with amino acid in RBD of $2009 \mathrm{H} 1$ are shown in Figure 3. The H-bond result shows that the all above 8 flavonoid compounds could form hydrogen bonding with key residues (Y98, N133, V135, T137, H183, D190, D225, E227 and G228) in binding pocket of HA. The structure alignment of SIA, ECG_13 and Puerarin 27 are shown in Figure 2(a) while their 2D structures are shown in Figure 2(b). Note that the ECG_13 and Puerarin_27 were well occupied in the active site of 2009 H1 in the orientation similar to those of SIA control structure. 
Table 1 The binding energy for most favorable complexes $(\mathrm{kcal} / \mathrm{mol})$ and percentage of possible conformation or dock score (\% DS).

\begin{tabular}{clccclcc}
\hline No. & \multicolumn{1}{c}{ Name } & $\begin{array}{c}\text { BE } \\
\text { (kcal/mol) }\end{array}$ & $\begin{array}{c}\text { \% } \\
\text { DS }\end{array}$ & NO. & \multicolumn{1}{c}{ Name } & $\begin{array}{c}\text { BE } \\
\text { (kcal/mol) }\end{array}$ & $\begin{array}{c}\text { \% } \\
\text { DS }\end{array}$ \\
\hline 1 & $3,5,7,4^{\prime}$-trimethoxyflavone & -6.19 & 76 & 19 & genistin & -5.16 & 24 \\
2 & $4 ', 5,7$-trimethoxyflavone & -6.02 & 52 & 20 & gossypetin & -5.99 & 71 \\
3 & $5,7,3^{\prime}, 4^{\prime}$-tetramethoxyflavone & -6.24 & 89 & 21 & hibiscetin & -6.33 & 62 \\
4 & 5,7 -dimethoxyflavone & -6.39 & 93 & 22 & kaempferol & -6.18 & 56 \\
5 & anthraquinone & -5.76 & 96 & 23 & kwakhurin & -6.94 & 39 \\
6 & catechin & -6.72 & 46 & 24 & malvidin & -6.84 & 95 \\
7 & catechin gallate (CG) & -7.63 & 30 & 25 & mirificin & -7.42 & 33 \\
8 & cyanidin & -6.76 & 43 & 26 & myricetin & -6.28 & 66 \\
9 & daidzein & -6.51 & 100 & 27 & puerarin & -7.89 & 46 \\
10 & daizin & -7.42 & 94 & 28 & quercetin & -6.21 & 45 \\
11 & delphinidin & -6.35 & 91 & 29 & quercetin-3- & -6.53 & 30 \\
& & & & & rhamnoside & & \\
12 & epicatechin (EC) & -6.73 & 85 & 30 & 2-methoxy-4-(2- & -4.31 & 63 \\
& & & & & propen-1-yl)phenol & & \\
13 & epicatechin gallate (ECG) & -7.20 & 80 & 31 & capsaisin & -6.08 & 27 \\
14 & epigallocatechin (EGC) & -6.74 & 77 & 32 & ferulicacid & -4.42 & 82 \\
15 & epigallocatechin gallate (EGCG) & -7.43 & 29 & 33 & ocoumaricacid & -4.70 & 85 \\
16 & catechin gallate (GC) & -7.25 & 61 & 34 & pcoumaricacid & -4.48 & 79 \\
17 & gallocatechin gallate (GCG) & -7.70 & 24 & 35 & protocetechuicacid & -4.15 & 73 \\
18 & genistein & -6.42 & 85 & & SIA (Control) & -4.68 & 99 \\
\hline
\end{tabular}
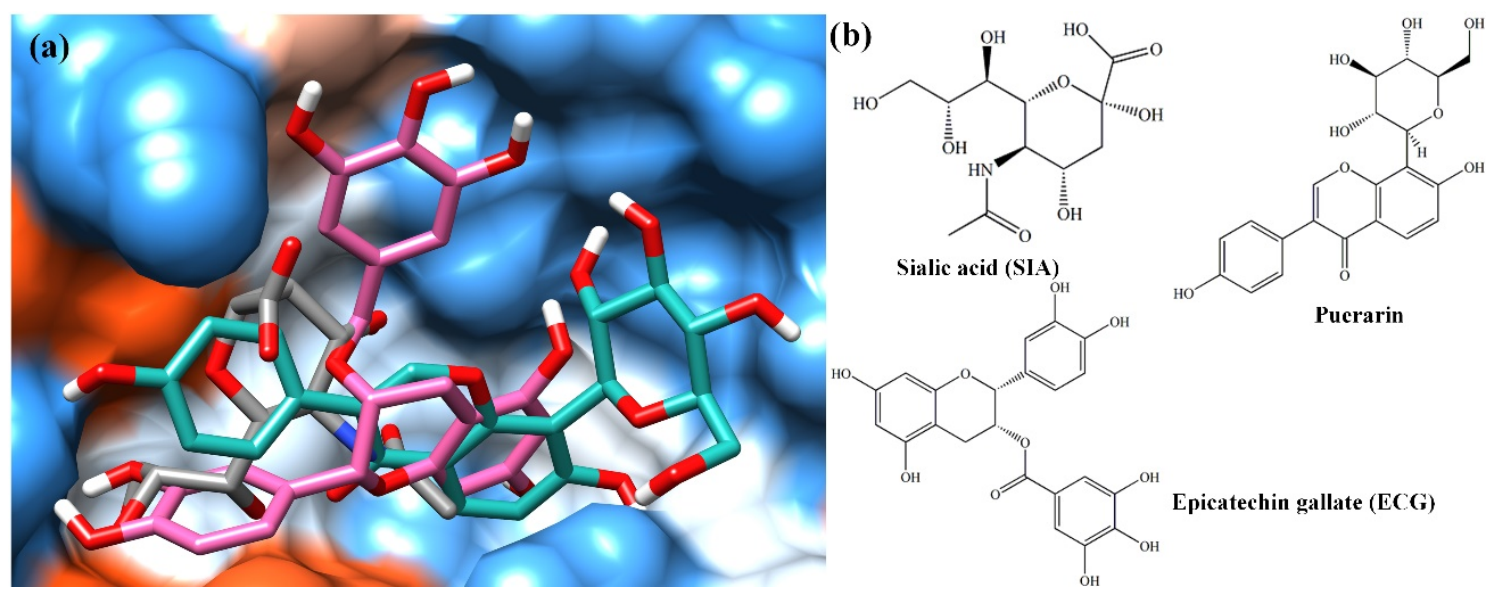

Figure 2 (a) The structure alignment of SIA (brown color), ECG_13 (pink color) and Puerarin_27 (blue color) where their 2D structures are shown in (b). 

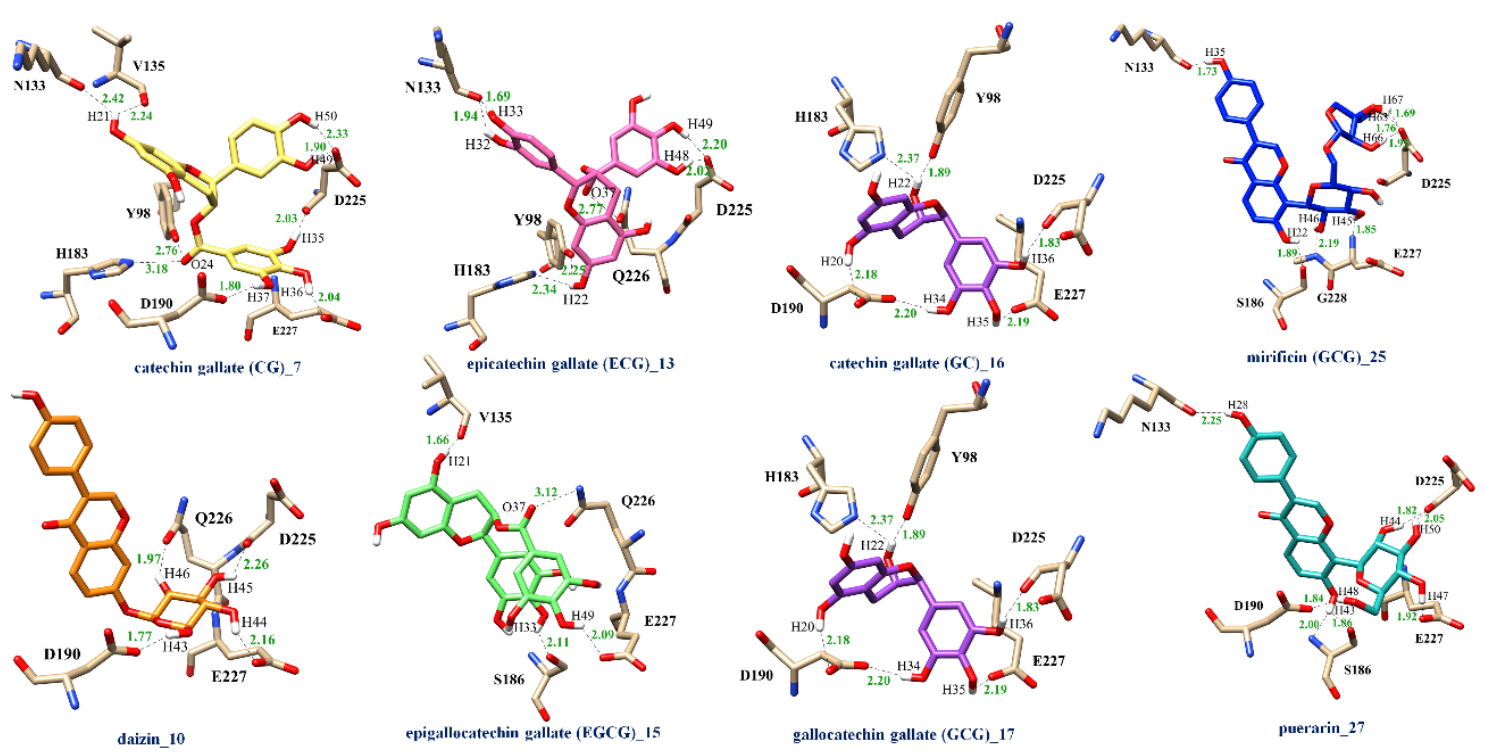

Figure 3 The hydrogen bond interaction and bond distances of 8 flavonoid compounds with amino acid in RBD of $2009 \mathrm{H} 1$.

The ECG_13, one of the major components of green tea, can inhibit HCV, HIV and influenza virus into host cell [8]. The docking results from Kannan et. al. in $2009 \mathrm{H} 1$ were quite similar to the present work in which the BE values of -catechin, epicatechin, EGC, ECG, EGCG are -7.0, -7.0, -7.1, -7.4 and 7.3 , respectively.

Puerarin, a major isofiavone compound isolated from the root of Pueraria lobate, had antioxidant, anti-inflammatory, antitumor, cholesterol lowering, liver protective, and neuroprotective properties. Additionally, few studies have explored the antiviral effect of puerarin and its target mechanism related to influenza virus. Wang et al. [40] reported that puerarin displayed an inhibitory effect on the potential inhibition of neuraminidase (NA) $\left(\mathrm{EC}_{50}=52.06 \mu \mathrm{M}\right)$. The docking energy of puerarin in binding pocket of $\mathrm{HA}$ in this work was $-7.89 \mathrm{kcal} / \mathrm{mol}$ closed to the previous study on NA binding pocket $(-8.8$ $\mathrm{kcal} / \mathrm{mol}$ ) [40], suggesting that puerarin displayed the binding susceptibility toward both NA and HA.

Therefore, the ECG_13 and the Puerarin_27 complexed with 2009 H1 were selected for molecular dynamics simulation study in order to focus on the structural and dynamical properties of the proteinligand complexes in the water medium and the hydrogen bonding and binding free energy of complexes.

\section{Molecular dynamics simulation results}

The docking studies provided valuable insight into possible interactions of the plant-based compounds with RBD of 2009 H1 HA protein, and only the best ranked and most populated conformation within the binding site was focused. To find further evidence of the binding of the ECG_13 and the Puerarin_27 to influenza hemagglutinin, MD simulation of H1-ECG and H1-Puerarin systems were performed in order to investigate the structural and dynamical properties of protein-ligand complexes in the water medium by using the AMBER 16 computational package.

\section{Stability of 2009 H1-ligand structures}

To investigate the stability of the 2 simulated systems, namely H1-ECG and H1-Puerarin, the root mean square displacements (RMSD) of protein, ligands and complexes for both complexes relative to the initial structure were evaluated along the $76 \mathrm{~ns}$ MD simulations (Figure 4). As the starting structure was taken from the molecular docking study, by the MD simulation both flavonoid compounds had the tendency to drift from their initial conformation for the structure relaxation to be better fitted in the binding pocket of $2009 \mathrm{H} 1 \mathrm{HA}$. RMSD plots of the complexes were fairly stable from $30-76$ ns as indicated by the small magnitude of RMSD fluctuation suggesting that H1- ECG and H1-Puerarin tended to reach an equilibrium state at $30 \mathrm{~ns}$. Therefore, the structural coordinates from the last $46 \mathrm{~ns}(31-76 \mathrm{~ns})$ simulations were collected as a production period for further analysis. 

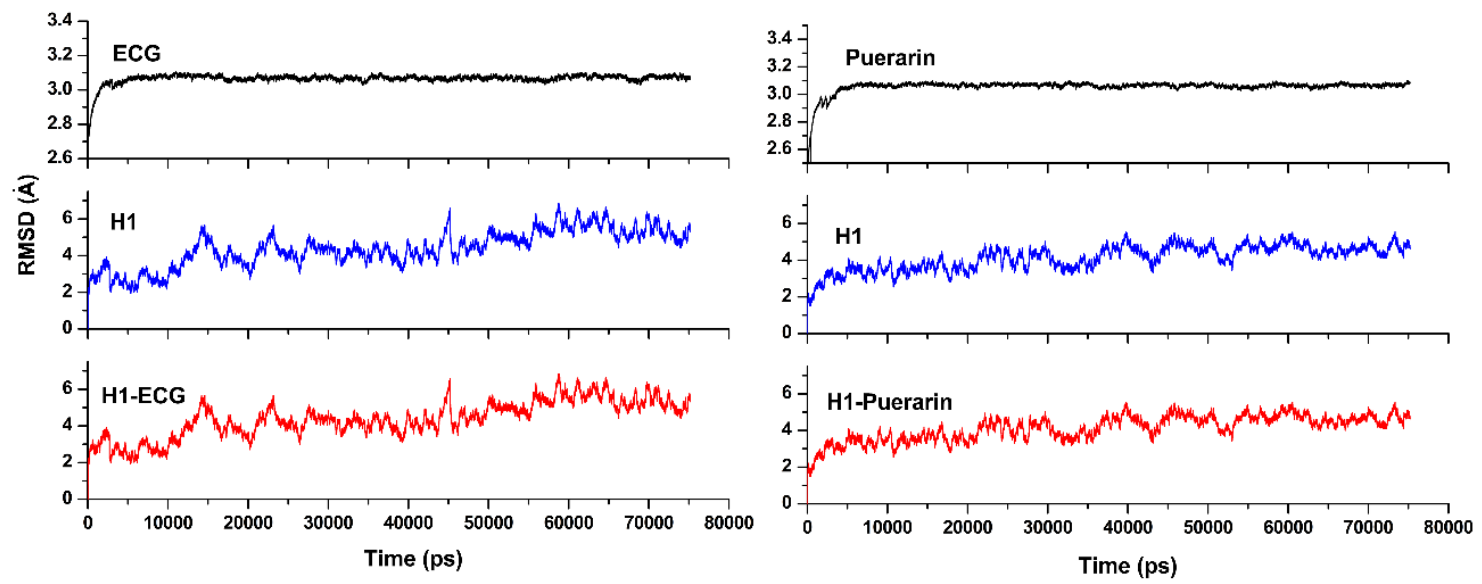

Figure 4 The root mean square displacements (RMSD) for the ligands (black line), 2009 H1 protein (blue line) and H1-ligand complexes (red line).

Hydrogen bonding and per-residue binding contribution of H1-ligand complexes

To gain insight into the efficiency of the ECG and Puerarin leading compounds binding to the 2009 H1 HA, the percentage of hydrogen bonds occupations between 2 ligands and the contact residues of the 2009 H1were measured according to the subsequent criteria: (i) the distance between proton donor (D) and acceptor (A) atoms of $\sim 3.5 \AA$ and (ii) the $\mathrm{D}-\mathrm{H}$...A angle of $\geq 120^{\circ}$. The analysis was carried out on the trajectories after equilibration. The calculated results are given in Table 2, while the hydrogen bond patterns of the ECG and Puerarin ligands in the binding pocket of 2009 H1 HA are depicted in Figure 4. Note that in aqueous solution, the hydrogen bond patterns from the MD simulation were different from the docking results, implying that in aqueous solution, both flavonoid compounds had adopted a new conformation to fit better within the active site of 2009 H1 HA.

Table 2 Percentage occupations for detected hydrogen bonds and decomposition energy (DC) between amino acid residues in $\mathrm{H} 1-\mathrm{ECG}$ and $\mathrm{H} 1-$ Puerarin complexes.

\begin{tabular}{|c|c|c|c|c|c|c|}
\hline \multirow[b]{2}{*}{ Regions } & \multicolumn{3}{|c|}{ H1- ECG } & \multicolumn{3}{|c|}{ H1-Puerarin } \\
\hline & Donor-Acceptor & $\begin{array}{l}\text { \% H-bond } \\
\text { Occupation }\end{array}$ & $\begin{array}{c}\text { DC } \\
\text { (kcal/mol) }\end{array}$ & Donor-Acceptor & $\begin{array}{l}\text { \% H-bond } \\
\text { Occupation }\end{array}$ & $\begin{array}{c}\text { DC } \\
(\mathrm{kcal} / \mathrm{mol})\end{array}$ \\
\hline $130-$ & OH16--O(V135) & 24.3 & -1.17 & (V135)N--O2 & 21.3 & -0.22 \\
\hline \multirow[t]{2}{*}{ loop } & OH16--O(V135) & 12.3 & & & & \\
\hline & OH8--O(S186) & 83.0 & -4.35 & (S186)N--O4 & 11.8 & -2.63 \\
\hline \multirow{3}{*}{$\begin{array}{l}\text { 190- } \\
\text { helix }\end{array}$} & (S186)N--O3 & 29.1 & & OH19--O(D190) & 59.9 & -5.21 \\
\hline & & & & OH19--O(D190) & 28.1 & \\
\hline & OH7--O(E227) & 26.9 & -8.93 & OH15--O(Q226) & 81.1 & -4.69 \\
\hline $220-$ & OH7--O(E227) & 19.7 & & OH18--O(E227) & 52.6 & -10.44 \\
\hline \multirow[t]{5}{*}{ loop } & & & & OH20--O(E227) & 47.3 & \\
\hline & & & & OH18--O(E227) & 45.5 & \\
\hline & & & & OH20--O(E227) & 29.0 & \\
\hline & & & & (E227)NH--O9 & 21.5 & \\
\hline & & & & (E227)NH--O6 & 16.8 & \\
\hline
\end{tabular}




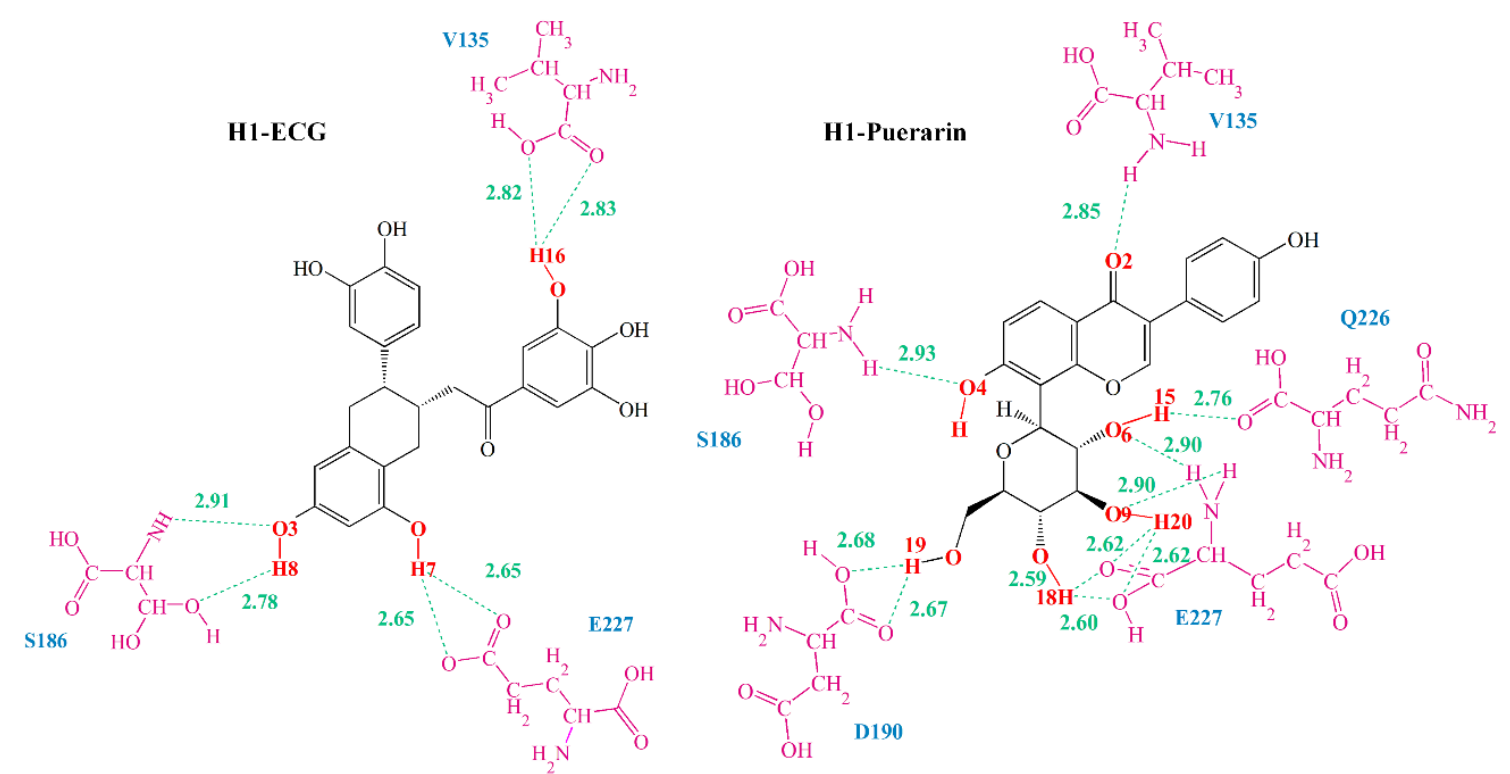

Figure 5 Hydrogen bond interaction of average structure of the H1- ECG and H1-Puerarin complexes obtained during the last $46 \mathrm{~ns}$ of MD simulations. Their distance $(\AA)$ were also labeled.

It can be seen from Figure 5 that the 2009 H1 residues located within $5 \AA$ of ligand provided larger contributions to the Puerarin binding at the active site than that of ECG. The main contributions for the H1- Puerarin complex were from the residues in the 130-loop (V135), 190-helix (S186 and D190) and 220-loop (Q226 and E227) regions. Among them, Q226 provided the one strongt hydrogen bond interaction toward hydroxy group $(\mathrm{OH} 1)$ of Puerarin with a bond length and \% occupations of $2.7576 \AA$ and 82.96, respectively. Interestingly, hydroxy group $(\mathrm{OH} 1, \mathrm{OH} 2$ and $\mathrm{OH} 3)$ of Puerarin form six hydrogen bonds with E227 at 220-loop with \% occupation of 52.6, 47.3, 45.5, 29.0, 21.5 and 16.8, respectively. Puerarin were also stabilized by 2 more hydrogen bonds with the V135, S186 backbone with $\%$ occupations of 21.3 and 11.8 , respectively and were also stabilized by 2 more hydrogen bonds with the D190 backbone with \% occupations of 59.92 and 28.13, respectively. This is in contrast for ECG compound in which one strong $(83.0 \%, 2.78 \AA)$ and one weak $(29.1 \%, 2.91 \AA)$ hydrogen bond with S186 at 190-helix region were detected. ECG were also stabilized by 2 more hydrogen bonds with the V135 backbone at 130-loop region (24.3 and $12.3 \%$ ) and were also stabilized by 2 more hydrogen bonds with the E227 side chain at 220-loop with \% occupations of 59.9 and 28.1, respectively.

Taking into account all the hydrogen bond data given above, the hydrogen bonds of the Puerarin ligand with key residues in the binding pocket of $2009 \mathrm{H} 1$ were found more than that of the ECG ligand. These hydrogen bond patterns seem to correspond with the binding affinities. From the computational findings, it can be suggested that the Puerarin ligand played a critical role by forming hydrogen bonding with key residues in the binding pocket of $2009 \mathrm{H} 1$ that may cause the higher $2009 \mathrm{H} 1$ inhibition.

To identify which residues of the HA are important for the flavonoid binding, the per-residue binding contribution between ligands and individual amino acids were calculated using the decomposition energy (DC) module of AMBER 16. The plot of the decomposition energies (DC) of those amino acids of 2009 H1, which are located within $5 \AA$ of the binding pocket, is shown in Table 2 and Figure 6(a). In addition, the van der Waals and electrostatic between ligands and those individual amino acids are also plotted in Figure 6(b) and Figure 6(c), respectively. Their interaction energies vary from -11 to 1 $\mathrm{kcal} / \mathrm{mol}$ for all systems. As can be seen in Figure 6, the interaction energies of $2009 \mathrm{H} 1$ with the both ligands are different. It is interesting to note that the E227 shows the largest contributions to the $2009 \mathrm{H1}$ ECG interaction energies, whilst the amino acids of $2009 \mathrm{H} 1$ with the highest interaction energies for Puerarin are Q226 and E227. From Figure 6(b) and Figure 6(c) confirmed that the main contribution interaction energy is come from electrostatic than that of van der Waals. This observation seems to be support to the previous section focused on hydrogen bonding where strong hydrogen bond with these amino acid residues could be observed. Therefore, the data leads us to conclude that the importance of these residues on the interaction energies is a primary source of inhibitors. 


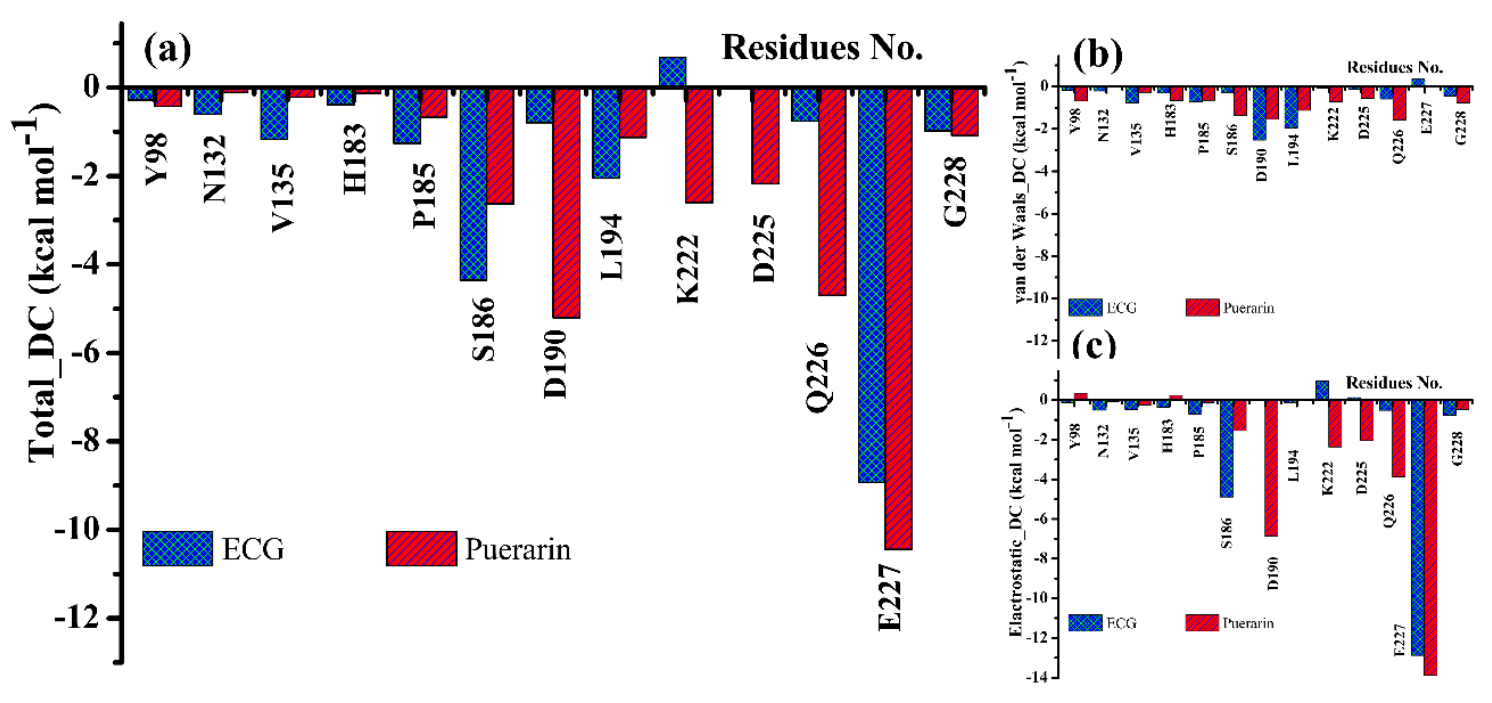

Figure 6 Total decomposition energy (a), van der Waals decomposition energy (b) and electrostatic decomposition energy (c) contribution of the H1- ECG and H1-Puerarin complexes.

The binding affinity free energy of 2009 H1-ligand complexes

To understand the binding efficiency of the Puerarin and ECG compounds within the binding pocket of $2009 \mathrm{H} 1$, the energy components and the averaged binding free energies of the complexes were calculated using MM-GBSA calculation. The electrostatic $\left(\Delta E_{e l e}\right)$, van der Waals $\left(\Delta E_{v d w}\right)$ and molecular mechanics $\left(\Delta E_{\mathrm{Mм}}\right)$ energies, non-polar $\left(\Delta G_{\text {sol }}^{\text {nonpolar }}\right)$ and polar solvation free energies, $\left(\Delta G_{\text {sol }}^{\text {ele }}\right)$ and entropic term $(-T \Delta S)$ of the 2 complexes were calculated from the 100 snapshots extracted from the last 40 ns. The energetics were summarized in Table 3 .

It can be seen from Table 4 that the electrostatic interactions appeared to be the major contribution for the $2009 \mathrm{H} 1$-ligands binding in accordance with the fact that the binding pocket of the $2009 \mathrm{H} 1$ was considerably hydrophilic. The electrostatic interactions of the H1-Puerarin $(-59.36 \pm 5.23 \mathrm{kcal} / \mathrm{mol})$ system was greater than that of H1-ECG $(-48.75 \pm 5.91 \mathrm{kcal} / \mathrm{mol})$ system. While the van der Waals interactions of the H1-Puerarin $(-30.03 \pm 2.58 \mathrm{kcal} / \mathrm{mol})$ system was slightly lesser than that of $\mathrm{H} 1-\mathrm{ECG}$ $(-32.31 \pm 3.34 \mathrm{kcal} / \mathrm{mol})$ system. This led to a stronger binding of Puerarin with $2009 \mathrm{H} 1 \mathrm{HA}(-89.39 \pm$ $7.81 \mathrm{kcal} / \mathrm{mol})$ over ECG $(-81.06 \pm 9.25 \mathrm{kcal} / \mathrm{mol})$ in gas phase. Concerning the structural change upon the simulation, the solvation contributions cannot be negligible for calculating the binding free energy of the complex. In addition, solvation term of the H1-Puerarin $63.53 \pm 4.24 \mathrm{kcal} / \mathrm{mol}$ ) system was higher than that of H1-ECG $(58.25 \pm 5.14 \mathrm{kcal} / \mathrm{mol})$ system. Therefore, when solvation term was included, the total binding free energy $\left(\Delta \mathrm{G}_{\text {binding }}\right)$ values of the H1-Puerarin $(-25.86 \pm 2.92 \mathrm{kcal} / \mathrm{mol})$ system was slightly greater than that of H1-ECG $(-22.81 \pm 2.19 \mathrm{kcal} / \mathrm{mol})$ system, suggesting that the Puerarin and ECG have the quite similar binding affinity towards 2009 H1 HA target. Although only one strong hydrogen bond with S186 was detected with ECG while more residues at the active site of HA (D187, Q226 and E227) could contribute with Puerarin ligand. The binding energy of puerarin with HA quite similar to the binding energy of puerarin with NA $(-19.49 \pm 3.37 \mathrm{kcal} / \mathrm{mol})$ that reported by Wang et al. [40]. The NA inhibition assay, puerarin exerted inhibitory effect on NA with $\mathrm{IC}_{50}$ values of $15.7 \mu \mathrm{M}$, while the $\mathrm{IC}_{50}$ value of oseltamivir was $89.01 \mu \mathrm{M}$ [40]. This information showed that puerarin could inhibit the activity of influenza virus.

Table 3 Energy components and binding energies (kcal/mol) of the H1-ECG and H1-Puerarin complexes.

\begin{tabular}{cccccccc}
\hline \multirow{2}{*}{ Parameters } & $\Delta E_{\text {ele }}$ & $\Delta \mathrm{E}_{\mathrm{vdW}}$ & $\Delta E_{\mathrm{мм}}$ & $\Delta G_{\text {sol }}^{\text {nonpolar }}$ & $\Delta G_{\text {sol }}^{\text {ele }}$ & $\Delta G_{\text {sol }}$ & $\Delta G_{\text {binding }}$ \\
\hline \multirow{2}{*}{ H1-ECG } & -48.75 & -32.31 & -81.06 & -5.33 & 63.58 & 58.25 & $\mathbf{- 2 2 . 8 1}$ \\
& \pm 5.91 & \pm 3.34 & \pm 9.25 & \pm 0.19 & \pm 5.19 & \pm 5.14 & $\pm \mathbf{2 . 1 9}$ \\
H1-Puerarin & -59.36 & -30.03 & -89.39 & -4.71 & 68.24 & 63.53 & $\mathbf{- 2 5 . 8 6}$ \\
& \pm 5.23 & \pm 2.58 & \pm 7.81 & \pm 0.15 & \pm 4.30 & \pm 4.24 & $\pm \mathbf{2 . 9 2}$ \\
\hline
\end{tabular}




\section{Conclusions}

In the present study, a set of flavonoids against 2009 H1N1 HA were screened by computational chemistry techniques. The docking results showed that the epicatechin gallate (ECG) and Puerarin exhibited a good binding affinity towards 2009 H1N1 HA. These 2 compounds were then studied by molecular dynamics (MD) simulations. The hydrogen bonds of the Puerarin ligand with key residues in the binding pocket of $2009 \mathrm{H} 1 \mathrm{HA}$ were found more than that of the ECG ligand. The electrostatic energy terms of $\mathrm{H} 1$-Puerarin system was greater than that of $\mathrm{H} 1-\mathrm{ECG}$ system. The binding free energy $\left(\Delta \mathrm{G}_{\mathrm{binding}}\right)$ values of the H1-Puerarin $(-25.86 \pm 2.92 \mathrm{kcal} / \mathrm{mol})$ system was slightly greater than that of H1-ECG ($22.81 \pm 2.19 \mathrm{kcal} / \mathrm{mol}$ ) system, suggesting that the Puerarin and ECG have the quite similar binding affinity towards $2009 \mathrm{H} 1 \mathrm{HA}$ target. The amino acids of $2009 \mathrm{H} 1$ that formed the highest interaction energy with Puerarin were Q226 and E227 residues while only S186 was detected the highest interaction energy with ECG. This information of ligand-protein interaction could be helpful in further drug design and development for influenza treatment.

\section{Acknowledgements}

This research was funded by National Higher Education, Science, Research and Innovation Policy Council, Thaksin University (Basic Research Fund; 64A105000028) Fiscal Year 2021. The authors would like to thank the Computational Chemistry Unit Cell, Faculty of Science, Chulalongkorn University, Thailand and the Department of Chemistry, Faculty of Science, Thaksin University, Thailand for providing research facilities, software packages and computing times.

\section{References}

[1] JJ Skehel and DC Wiley. Influenza viruses and cell membranes. Am. J. Respir. Crit. Care Med. 1995; 152, S13-S15.

[2] T Lin, G Wang, A Li, Q Zhang, C Wu, R Zhang, Q Cai, W Song and KY Yuen. The hemagglutinin structure of an avian H1N1 influenza A virus. Virology 2009; 392, 73-81.

[3] DA Steinhauer. INFLUENZA pathways to human adaptation. Nature 2013; 499, 412-3.

[4] DA Steinhauer. Role of hemagglutinin cleavage for the pathogenicity of influenza virus. Virology. 1999; 258, 1-20.

[5] Y Su, L Meng, J Sun, W Li, L Shao, K Chen, D Zhou, F Yang and F Yu. Design, synthesis of oleanolic acid-saccharide conjugates using click chemistry methodology and study of their antiinfluenza activity. Eur. J. Med. Chem. 2019; 182, 111622.

[6] Z Jin, Y Wang, XF Yu, QQ Tan, SS Liang, T Li, H Zhang, PC Shaw, J Wang and C Hu. Structurebased virtual screening of influenza virus RNA polymerase inhibitors from natural compounds: Molecular dynamics simulation and MM-GBSA calculation. Comput. Biol. Chem. 2020; 85, 107241.

[7] H Li, M Li, R Xu, S Wang, Y Zhang, L Zhang, D Zhou and S Xiao. Synthesis, structure activity relationship and in vitro anti-influenza virus activity of novel polyphenol-pentacyclic triterpene conjugates. Eur. J. Med. Chem. 2019; 163, 560-68.

[8] S Onishi, T Mori, H Kanbara, T Habe, N Ota, Y Kurebayashi and T Suzuki. Green tea catechins adsorbed on the murine pharyngeal mucosa reduce influenza A virus infection. J. Funct. Foods. 2020; 68, 103894.

[9] C Seniya, S Shrivastava, SK Singh, GJ Khan. Analyzing the interaction of a herbal compound Andrographolide from Andrographis paniculata as a folklore against swine flu (H1N1). Asian Pacific J. Trop. Dis. 2014; 4, S624-S630.

[10] R Latif and CY Wang. Andrographolide as a potent and promising antiviral agent. Chin. J. Nat. Med. 2020; 18, 760-9.

[11] WC Hsu, SP Chang, LC Lin, CL Li, CD Richardson, CC Lin and LT Lin. Limonium sinense and gallic acid suppress hepatitis $\mathrm{C}$ virus infection by blocking early viral entry. Antiviral Res. 2015; 118, 139-47.

[12] BS Hwang, IK Lee, HJ Choi and BS Yun. Anti-influenza activities of polyphenols from the medicinal mushroom Phellinus baumii. Bioorg. Med. Chem. Lett. 2015; 25, 3256-60.

[13] R Fioravanti, I Celestino, R Costi, GC Crucitti, L Pescatori, L Mattiello, E Novellino, P Checconi, AT Palamara, L Nencioni and RD Santo. Effects of polyphenol compounds on influenza A virus replication and definition of their mechanism of action. Bioorgan. Med. Chem. 2012; 20, 5046-52. 
[14] S Kannan and P Kolandaivel. Antiviral potential of natural compounds against influenza virus hemagglutinin. Comput. Biol. Chem. 2017; 71, 207-18.

[15] L Meng, Y Su, F Yang, S Xiao, Z Yin, J Liu, J Zhong, D Zhou and F Yu. Design, synthesis and biological evaluation of amino acids-oleanolic acid conjugates as influenza virus inhibitors. Bioorgan. Med. Chem. 2019; 27, 115147.

[16] YT Wang, CH Chan, ZY Su and CL Chen. Homology modeling, docking, and molecular dynamics reveal HR1039 as a potent inhibitor of 2009 A(H1N1) influenza neuraminidase. Biophy. Chem. 2010; 147, 74-80.

[17] P Kongsune, S Hannongbua. The role of conserved QXG and binding affinity of S23G \& S26G receptors on avian $\mathrm{H} 5$, swine $\mathrm{H} 1$ and human $\mathrm{H} 1$ of influenza A virus hemagglutinin. J. Mol. Graph. Model. 2018; 82, 12-19.

[18] FC Bernstein, TF Koetzle, GJ Williams, EFJ Meyer, MD Brice, JR Rodgers, O Kennard, T Shimanouchi, M Tasumi. The Protein Data Bank: A computer-based archival file for macromolecular structures. J. Mol. Biol. 1977; 112, 535-42.

[19] R Xu, R McBride, CM Nycholat, JC Paulson, IA Wilson. Structural Characterization of the Hemagglutinin Receptor Specificity from the 2009 H1N1 Influenza Pandemic. J. Virol. 2012; 86, 982-90.

[20] R Dennington, T Keith and J Millam. GaussView. Semichem Inc., Shawnee Mission KS., 2009.

[21] MJ Frisch, M Frisch, G Trucks, K. Schlegel, G Scuseria, M Robb, J Cheeseman, J Montgomery, T Vreven, KN Kudin, J Burant, J Millam, S Iyengar, J Tomasi, V Barone, B Mennucci, M Cossi, G Scalmani, N Rega, A Petersson, H Nakatsuji, M Hada, M Ehara, K Toyota, R Fukuda, J Hasegawa, M Ishida, T Nakajima, Y Honda, O Kitao, H Nakai, M Klene, X Li, J Knox, H Hratchian, D Cross, V Bakken, C Adamo, J Jaramillo-Merchan, R Gomperts, R Stratmann, O Yazyev, A Austin, R Cammi, C Pomelli, J Ochterski, P Ayala, K Morokuma, G Voth, P Salvador, JJ Dannenberg, VG Zakrzewski, S Dapprich, AD Daniels, M Strain, O Farkas, S Malick, A Rabuck, K Raghavachari, J Foresman, J Ortiz, Q Cui, AG Baboul, S Clifford, J Cioslowski, B Stefanov, G Liu, A Liashenko, P Piskorz, I Komaromi, R Mata, D Fox, T Keith, S Laham, CY Peng, A Nanayakkara, M Challacombe, P Gill, B Johnson, W Chen, M Wong, RS González, J Pople, J Dannenberg, V Zakrzewski, A Daniels, AG Baboul, Y Peng, GE Scuseria, JM Millam, JB Foresman, MJ Frisch, JA Montgomery, RE Stratmann, DK Malick, XB Li, T Keith, MW. Wong, HB Schlegel, PY Ayala, BB Stefanov, M Hada, RL Martin, KN Kudin, HP Hratchian, GA Voth, AJ Austin, MC Strain, C Adamo, PMW Gill, MA Robb, GA Petersson, JB Cross, JL Torre, GW Trucks, JC Burant, DJ Fox,bAD Rabuck, C Huerta, M Akhras, JR Cheeseman, SS Iyengar, JA Pople, JE Knox, JW Ochterski and BA Johnson. Gaussian 03, revision C.02, Gaussian, Inc., Wallingford CT, 2004.

[22] GM Morris, DS Goodsell, RS Halliday, R Huey, WE Hart, RK Belew and AJ Olson. Automated docking using a Lamarckian genetic algorithm and empirical binding free energy function. $J$. Comput. Chem. 1998; 19, 1639-62.

[23] J Gasteiger and M Marsili. Iterative partial equalization of orbital electronegativity-a rapid access to atomic charges. Tetrahedron 1980; 36, 3219-28.

[24] GM Morris, R Huey, W Lindstrom, MF Sanner, RK Belew, DS Goodsell and AJ Olson. AutoDock4 and AutoDockTools4: Automated docking with selective receptor flexibility. J. Comput. Chem. 2009; 30, 2785-91.

[25] N Nunthaboot, T Rungrotmongkol, M Malaisree, N Kaiyawet, P Decha, P Sompornpisut, Y Poovorawan and S Hannongbua. Evolution of human receptor binding affinity of H1N1 hemagglutinins from 1918 to 2009 pandemic influenza A virus. J. Chem. Inf. Model. 2010; 50, 141017.

[26] R Huey, GM Morris, AJ Olson and DS Goodsel. A semiempirical free energy force field with charge-based desolvation. J. Comput. Chem. 2007; 28, 1145-52.

[27] E Ermakova. Structural insight into the glucokinase-ligands interactions: Molecular docking study. Comput. Biol. Chem. 2016; 64, 281-96.

[28] D Case, R Betz, DS Cerutti, T Cheatham, T Darden, R Duke, TJ Giese, H Gohlke, A Götz, N Homeyer, S Izadi, P Janowski, J Kaus, A Kovalenko, TS Lee, S LeGrand, P Li, C Lin, T Luchko and P Kollman. Amber 16. University of California, San Francisco, 2016.

[29] WD Cornell, P Cieplak, CI Bayly and PA Kollmann. Application of RESP charges to calculate conformational energies, hydrogen bond energies, and free energies of solvation. J. Am. Chem. Soc. 1993; 115, 9620-31. 
[30] Y Duan, C Wu, S Chowdhury, MC Lee, G Xiong, W Zhang, R Yang, P Cieplak, R Luo, T Lee, J Caldwell, J Wang and P Kollman. A point-charge force field for molecular mechanics simulations of proteins based on condensed-phase quantum mechanical calculations. J. Comput. Chem. 2003; 24, 1999-2012.

[31] WD Cornell, P Cieplak, CI Bayly, IR Gould, KM Merz, DM Ferguson, DC Spellmeyer, T Fox, JW Caldwell and PA Kollman. A second generation forcefield for the simulation of proteins, nucleicacids, and organic-molecules. J. Am. Chem. Soc. 1995; 117, 5179-97.

[32] JM Wang, W Wang and PA Kollman. Antechamber: An accessory software package for molecular mechanical calculations. J. Am. Chem. Soc. 2001; 222, U403-U403.

[33] DC Bas, DM Rogers and JH Jensen. Very fast prediction and rationalization of pKa values for protein-ligand complexes. Proteins 2008; 73, 765-83.

[34] $\mathrm{H} \mathrm{Li}, \mathrm{AD}$ Robertson and JH Jensen. Very fast empirical prediction and rationalization of protein pKa values. Proteins. 2005; 61, 704-21.

[35] HJC Berendsen, JPM Postma, WF Van Gunsteren, A DiNola and JR Haak. Molecular dynamics with coupling to an external bath. J. Chem. Phys. 1984; 81, 3684-90.

[36] JP Ryckaert, G Cicotti and HJC Berendsen. Numerical integration of the Cartesian equations of motion of a system with constraints: Molecular dynamics of n-alkanes. J. Comput. Phys. 1977; 23, 327-41.

[37] DM York, TA Darden and LG Pedersen. The effect of long-range electrostatic interactions in simulations of macromolecular crystals: a comparison of the Ewald and truncated list methods. $J$. Chem. Phys. 1993; 99, 8345-8.

[38] DR Roe and TE Cheatham. PTRAJ and CPPTRAJ: Software for processing and analysis of molecular dynamics trajectory data. J. Chem. Theory. Comput. 2013; 9, 3084-95.

[39] N Nunthaboot, T Rungrotmongkol, M Malaisree, P Decha, N Kaiyawet, P Intharathep, P Sompornpisut, Y Poovorawan, S Hannongbua. Molecular insights into human receptor binding to 2009 H1N1 influenza A hemagglutinin. Monatsh Chem. 2009; 141, 801-7.

[40] HX Wang, MS Zeng, Y, Ye, JY Liu and PP Xu. Antiviral activity of puerarin as potent inhibitor of influenza virus neuraminidase. Phytother. Resh. 2021; 35, 324-36. 